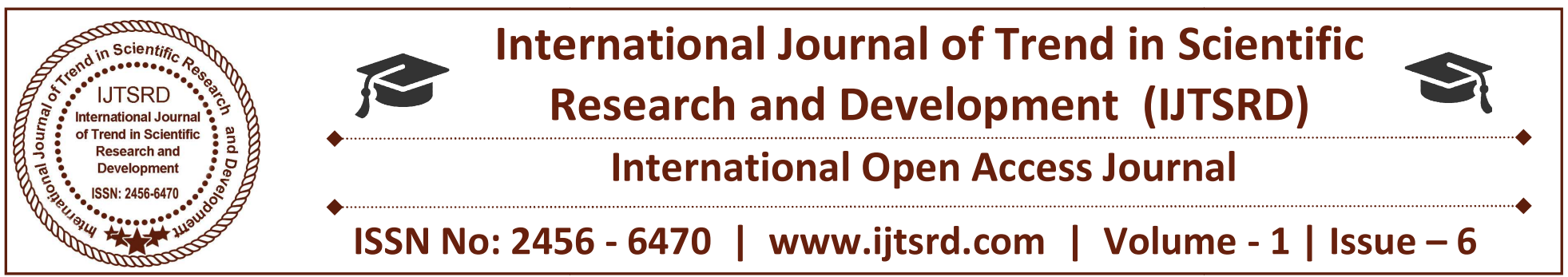

\title{
New Advancements in the Applications of Fractional Calculus in Science and Engineering
}

\author{
Savita Sharma \\ Department of Mathematics, \\ Maharishi Arvind International Institute of Technology, \\ Kota, Rajasthan, India
}

\begin{abstract}
Fractional Calculus is a study of an extension of derivatives and integrals to non-integer orders and also linking its origins with classical integral and differential calculus. The interesting part of this subject is that fractional derivatives and integrals are not a local or point property or quantity. In some few years considerable interest in fractional calculus has been seen by the applications it finds in various areas of engineering, science, applied mathematics, finance and bio-engineering as possibly it includes fractal phenomena too. This paper deals with the researchers of engineering and science who are learning about Fractional Calculus and its possible applications in their fields of study. The paper focuses on the review of new growth based on the fractional calculus in different fields both on theoretical and application facets.
\end{abstract}

Keywords: Fractional calculus, fractional order, integral and differential, modeling, dynamics

\subsection{Introduction}

During the second half of the twentieth century, considerable amount of research in fractional calculus was published in engineering literature. As a matter of fact, in this recent advances of fractional calculus which are dominated by modern examples of applications in differential and integral equations, fluid mechanics, viscoelasticity, mathematical biology, physics, signal processing and electrochemistry. Undoubtly fractional calculus has become an exciting new mathematical method of solution of diverse problems in mathematics, science, and engineering. In order to stimulate more interest in the subject and to show its utility, this paper is devoted to new and recent applications of fractional calculus in science and engineering.

If we see back in history Integration and differentiation to an arbitrary order named fractional calculus [1, 2, and 3]. The concept of non-integral order of integration can see its footprint of the genesis of differential calculus itself: the philosopher and creator of modern calculus, the Newton's rival Leibniz made some remarks on the meaning and possibility of fractional derivative of order $1 / 2$ in the late of 17-th century. However, a countinuos work was first carried out by Liouville in a serious of papers from 1832-1837, where he defined the first outcast of an operator of fractional integration. Initially before fractional operations, we see generalization of integer order calculus to an arbitrary order, class of fractionally differentiable functions (called "differ integrable functions" see, [1, 2, 3], where use of the term is widely discussed) and applications of the calculus.

The study of fractional calculus has applications in varied and comprehensive fields of engineering and science such as electromagnetics, fluid mechanics, electrochemistry, viscoelasticity biological population models, optics, and signals processing. In this many 
application this fractional calus has been modeled to physical and engineering processes that are found to be best described by fractional differential equations. In order to require accurate modelling of damping fractional derivative models are used for accurate modelling. In these fields, various analytical analysis method and numerical methods including their applications to new problems have been used in some years. We can more say that fractional calculus is a bit tricky to interpret, seeming at first to be a weird generalisation of calculus .Certainly, there is some magic hidden behind fractional calculus!

In the last years has found use in studies of viscoelastic materials, as well as in many fields of science and engineering including fluid flow, rheology, diffusive transport, electerical networks, electromagnetic theory and probability. In this paper we consider different definitions of fractional derivatives and integrals (differintegrals). Also we present some applications of fractional calculus in science and engineering.

\subsection{Fractional Calculus}

Fractional calculus is a branch of mathematical analysis that studies the possibility of taking real number, or even complex number, powers of the differential operator

$$
D=\frac{d}{d x}
$$

and the integration operator J.(Usually $\mathrm{J}$ is used in favor of I to avoid with other I-like identities)

In this context powers refer to iterative application or composition, in the same sense that

$$
f^{2} x=f(f(x))
$$

For example, one may pose the question of interpreting meaningfully $\quad \sqrt{D}=D^{1 / 2}$ as a square root of the differentiation operator (an operator half iterate), i.e., an expression for some operator that when applied twice to a function will have the same effect as differentiation. We are all familiar with the idea of derivatives.

The usual notation:

$$
\frac{d f(x)}{d x}=D^{1} f(x), \frac{d^{2} f(x)}{d x}=D^{2} f(x)
$$

also properties like

$$
D^{1}[f(x)+g(x)]=D^{1} f(x)+D^{1} g(x)
$$

But what would be the meaning of notation like

$$
\frac{d^{1 / 2} f(x)}{d x^{1 / 2}}=D^{1 / 2} f(x) ?
$$

The notion of derivative of order $1 / 2$ (fractional derivative) was discussed briefly as early as the XVIII century by Leibniz. Other giants of the past including L'Hopital, Euler, Lagrange, Laplace, Riemann, Fourier, Liouville, and others toyed with the idea.

Riemann-Liouville definition of fractional order differ-integral:

$$
R L_{\alpha} D_{t}^{\alpha} f(t)=\frac{1}{\Gamma(\mathrm{m}-\alpha)} \frac{d^{m}}{d t} \int_{\alpha}^{t}(t-\tau)^{m-\alpha-1} f(\tau) d \tau
$$

Where $m-1<\alpha \leq m \in N$

and $\alpha \in \mathrm{R}$ ( $\mathrm{R}$ is the set of real numbers) is a fractional order of the differ-integral of the function $f(t)$.

After that mathematicians like Fourier, Euler, Laplace have studied fractional calculus (integration and differentiation) and its mathematical consequences. The order of the fractional derivative $\alpha$ where $0<\alpha<1$ classifies and quantifies the influence of past history. Note that fractional derivative definitions have inbuilt integration, meaning that the operation is non-local, requires information about past states. For unity value of the fractional order (or integer of order one) the influence of past history is minimal, in the sense that, then the process evolution enters only through the present state. Small value of $\alpha$ corresponds to a strong influence of past history.

Many authors have developed and defined the concept of fractional integrals and derivatives (often called differ integrals) in their own way. Of them, the name of Riemann- Liouville, Weyl and Grunwald-Letnikov are worth mentioning. However, Caputo reformulated the more "classic" definition of the RiemannLiouville fractional derivative in order to use integer order initial condition to solve fractional order differential equation. It is in 1996, Kolwankar and Gangal reformulated again, the Riemann-Liouville fractional derivative in order to differentiate nowhere differentiable fractal functions. Several applications and physical manifestations of fractional calculus related to science and technology have been found in 20th century. The applications like, Abel's fractional integral equation of Tatochrome, Modelling of speech signals, Modelling of cardiac tissue electrode interface, Fractional differentiation of edge detection related to image processing, the solution of time- 
dependent viscous- diffusion fluid problems are worth mentioning. Some other applications are sound waves propagation in rigid porous materials, lateral and longitudinal control of autonomous vessels, ultrasonic wave propagation in human cancellous bone, applications in viscous elasticity, polymer science, fractal phenomena .

Several analytical methods are used to solve fractional order differential equation. The Laplace transform method is a very popular technique although other analytical techniques do exist. Some more analytical methods are Mellin's transform, power series expansion, method approach using Green's function, Babenko's symbolic method, complementary orthogonal polynomial method, Reisz fraction potential method etc. In this connection several functions of fractional calculus viz., Beta functions, Gamma functions, Mittag-Leffer function, Miller Ross function, Agarwal function etc. is addressed . However, a very new approach to solve fractional differential equation is Adomain Decomposition Method (ADM). In this paper, we solve a fractional differential equation of mass spring system using ADM.

\subsection{Fractional Derivative}

As far as the existence of such a theory is concerned, the foundations of the subject were laid by Liouville in a paper from 1832. The fractional derivative of a function to order is often now defined by means of the Fourier or Mellin integral transforms. An important point is that the fractional derivative at a point $\mathrm{x}$ is a local property only when a is an integer; in noninteger cases we cannot say that the fractional derivative at $x$ of a function $f$ depends only on the graph of $f$ very near $x$, in the way that integer-power derivatives certainly do. Therefore it is expected that the theory involves some sort of boundary conditions, involving information on the function further out. To use a metaphor, the fractional derivative requires some peripheral vision.

\subsection{Applications in applied sciences and engineering}

Fractional order calculus can represent systems with high-order dynamics and complex nonlinear phenomena using few coefficients, since the arbitrary order of the derivatives provides an additional degree of freedom to fit a specific behavior. Another important characteristic is that fractional order derivatives depend not only on local conditions of the evaluated time but also on the entire history of the function. This fact is often useful when the system has a long-term "memory" and any evaluation point depends on the past values of the function. At this point we therefore consider it relevant to present some applications involving the implementation of FOCbased models in different physical systems, namely: the diffusion equation, food engineering, robotics and control theory, and econophysics.

\subsubsection{Applications to transport in fusion plasmas}

Here we present phenomenological models of plasma transport based on the use of fractional diffusion operators. In particular, we extend the fractional diffusion models discussed before, by incorporating finite-size domain effects, boundary conditions, sources, spatially dependent diffusivities, and general asymmetric fractional operators. These additions are critical in order to order to go beyond tracers transport calculations. We show that the extended fractional model is able to reproduce within a unified framework some of the phenomenology of non-local, non diffusive transport processes observed in fusion plasmas, including anomalous confinement time scaling, "up-hill" transport, pinch effects, and on-axis peaking with off-axis fuelling.

\subsubsection{Application to reaction diffusion systems}

Here we discuss the role of fractional diffusion in reaction-diffusion systems. In particular, we present a numerical and analytical study of front propagation in the fractional Fisher-Kolmogorov equation.

\subsubsection{Fractional-order model of neurons in biology}

Robinson [4] described the neurodynamics of the vestibulo-ocular reflex (VOR) model. The main function of VOR is to keep the retinal image stable by producing eye rotations which counter balance head rotations. The fractional-order dynamics of vestibulooculomotor neurons suggests that fractional-order rather than integer-order forms of signal processing occur in the vestibulo-oculomotor system.

\subsubsection{Fractional calculus in electro chemistry and tracer fluid flows}

Although the idea of a half-order fractional integral of the current field was known in electrochemistry, Oldham [5] has initiated a mathematical study of some semi-integral electro analysis with some 
experimental support. Simultaneously the authors (Oldham and Spanier [6]) have given considerable attention to their new approach to the solution of electrochemical problems that deal with diffusion phenomena. Subsequently, Goto and Ishii [7] developed the idea of semi differential electro analysis with the fractional-order diffusion equation that may occur in other fields including diffusion, heat conduction, and mass transfer.

\subsubsection{Electrical circuits with fractance}

Classical electrical circuits consist of resistors and capacitors and are described by integer-order models. However, circuits may have the so-called fractance which represents an electrical element with fractionalorder impedance as suggested in the paper by Le Mehaute and Crepy [8].

\subsubsection{Fractional-order dynamical systems in control theory.}

New and effective methods for the time-domain analysis of fractional-order dynamical systems are required for solving problems of control theory. As a new generalization of the classical PID-controller, the idea of PI $\lambda \mathrm{D} \mu$-controller, involving fractional-order integrator and fractional-order differentiator, has been found to be a more efficient control of fractional-order dynamical systems.

\subsubsection{Generalized voltage divider}

Both the tree fractance and chain fractance consist not only of resistors and capacitors properties, but also they exhibit electrical properties with nonintegerorder impedance. He generalized the classical voltage divider in which the fractional order impedances F1 and F2 represent impedances not only on Westerlund's capacitors, classical resistors, and induction coils, but also impedances of tree fractance and chain fractance.

\subsubsection{Fractional calculus in Robotics}

In robotics, calculus has a vital role in defining robot motion such as forward and backward movement, rotation, path and trajectory planning, velocity etc; robot control such as force control, multivariable control, vision based control etc and robot vision such as geometry of image formation, camera calibration etc. Fractional calculus has been shown to improve and generalized well established control methods. Fractional order controller was introduced by Oustaloup [9], who developed CRONE controller in pursuing fractal robustness.

\subsubsection{Fractional Calculus in Analytical Science}

Fractional calculus has recently been shown useful in areas such as milk adulteration [10], ghee adulteration [11] etc. Milk adulteration is an important issue, economically and as well as health point of view. In literature, there have been developed many methods to detect the adulteration of milk. In $[10,12]$, electrical admittance spectroscopy has been used for detecting fat and water content in milk and freezing point osmometryof milk scheme has been used for measuring the concentration of water in [13].

\subsubsection{Fractional Differentiation based image processing}

Fractional calculus is recently being shown to be useful in various image processing techniques such as edge detection[14] and texture segmentation, revealing faint objects[15,16], image restoration etc. In paper author [17] have shown how to remove the incoherent light scattering produced by a random medium from image, using fractional Fourier Transform. Results have shown substantial improvement in quality of astronomical images. Edge detection often make use of integer order differentiation operators, order 1 used by the gradient and order 2 used by the Laplacian. In [14], authors have shown the advantage of fractional order derivatives to improve the criteria of thin detection and criteria of immunity to noise. Texture Enhancement of medical images is being proposed in [18] using fractional differential mask. Results show that the fractional differential operator can extract subtle information thus improving textual performance.

\subsubsection{Ultrasonic wave propagation in human cancellous bone}

Fractional calculus is used [19] to describe the viscous interactions between fluid and solid structure. Reflection and transmission scattering operators are derived for a slab of cancellous bone in the elastic frame using Blot's theory. Experimental results are compared with theoretical predictions for slow and 
fast waves transmitted through human cancellous bone samples.

\subsubsection{Using Fractional Calculus for Lateral and Longitudinal Control of Autonomous Vehicles}

The use of Fractional Order Controllers (FOC) applied to the path-tracking problem in an autonomous electric vehicle[20]. A lateral dynamic model of a industrial vehicle has been taken into account to implement conventional and Fractional Order Controllers. Several control schemes with these controllers have been simulated and compared.

\section{CONCLUSION}

In this paper given a basic introduction to fractional calculus and its application in various domains. We first introduce some basic definitions and formulae which are used in the paper and discussed some application. We have shown how fractional calculus can add more expressive power to the existing integer models, by analyzing mortgage problem and fractional oscillator. To motivate further research, we introduce the state-of-the-art of fractional calculus and its applicability in various engineering and science domains.

\section{REFERENCES}

[1] K.B. Oldham and J. Spanier, The Fractional Calculus, Academic Press, New YorkLondon, 1974.

[2] S. G. Samko, A. A. Kilbas and O. I. Maritchev, Integrals and Derivatives of the Fractional Order and Some of their Applications, [in Russian], Nauka i Tekhnika, Minsk, 1987.

[3] A.J. Turski, B, Atamaniuk and E. Turska, Principles of Fractional Calculus, Integro-Derivatives of Arbitrary Order, (in Polish), ITR Reports, 2/2004

[4] D. A. Robinson, The use of control systems analysis in neurophysiology of eye movements, Ann. Rev. Neurosci. 4 (1981), 462-503.

[7] M. Goto and D. Ishii, Semidifferential electroanalysis, J. Electroanal. Chem. and Interfacial Electrochem. 61 (1975), 361-365.

[8] A. Le Mehaute and G. Crepy, Introduction to transfer and motion in fractal media: the geometry of kinetics, Solid State Ionics 9-10 (1983), 17-30.
[5] K. B. Oldham, A signal-independent electroanalytical method, Anal. Chem. 44 (1972), no. 1, 196-198.

[6], The Fractional Calculus, Mathematics in Science and Engineering, vol. 111, Academic Press, New York, 1974.

[9] A. Oustaloup, La Commande CRONE: CommandeRobusted ${ }^{\text {ee }}$ Ordre Non Entier, Editions Hermès, Paris, 1991.

[10] Mabrook, M. F., and M. C. Petty. "A novel technique for the detection of added water to full fat milk using single frequency admittance measurements." Sensors and Actuators B: Chemical 96.1, pp 215218, 2003.

[11] Farag, R. S. "Fractional crystallization and gaschromatographie analysis of fatty acids as a means of detectingbutterfat adulteration."Journal of the American Oil Chemists Society, 1665-1669,1983.

[12] Mabrook, M. F., and M. C. Petty. "Application of electrical admittance measurements to the quality control of milk." Sensors and Actuators B: Chemical, 136-141,2002.

[13] Büttel, Britta, Markus Fuchs, and Birger Holz. "Freezing point osmometry of milk to determine the additional water content-an issue in general quality control and German food regulation." Chemistry Central Journal, 2008.

[14] B. Mathieu, P. Melchior, A. Oustaloup, Ch. Ceyral, Fractional differentiation for edge detections, Signal Processing, pp.2421-2432, 2003. [15] JiaHuading and $\mathrm{Pu}$ Yifei, Fractional calculus method for enhancing digital image of bank slip, Proceedings of the 2008 Congress on Image and Signal Processing, Vol. 3, pp.326-330,2008.

[16] Y. Pu, W.X. Wang, J.L. Zhou, Y.Y. Wand and H.D. Jia, Fractional differential approach to detecting textural features of digital image and its fractional differential filter implementation, Sci. China Ser. F Inf. Sci., 51(9), pp.1319-1339,2008.

[17] J.M. Blackledge, Diffusion and fractional diffusion based image processing, EG UK Theory and Practice of Computer Graphics, pages: 233 - 240, Cardiff, 2009.

[18] Hamid A. Jalab and Rabha W. Ibrahim, "Texture Enhancement for Medical Images Based on Fractional 
Differential Masks," Discrete Dynamics in Nature and Society, vol. 2013, 2013.

[19] N. Sebaa, Z. E. A. Fellah, W. Lauriks, C. Depollier, Application of fractional calculus to ultrasonic wave propagation in human cancellous bone, Signal Processing archive Volume 86 , Issue 10 (2006)2668 - 2677

[20] J.I. Sua'rez , B.M. Vinagre , A.J. Calder'on , C.A. Monje and Y.Q. Chen Using Fractional Calculus for Lateral and Longitudinal Control of Autonomous Vehicles Lecture Notes in Computer Science, Springer Berlin / Heidelberg, Volume 2809/2004 